\title{
Fragmenting gadolinium: Mononuclear polyoxometalate-based magnetic coolers for ultra-low temperatures
}

By María-José Martínez-Pérez, Oscar Montero, Marco Evangelisti,* Fernando Luis, Javier Sesé, Salvador Cardona-Serra, and Eugenio Coronado*

[*] Dr. M. J. Martínez, Dr. O. Montero, Dr. M. Evangelisti, Dr. F. Luis

Instituto de Ciencia de Materiales de Aragón (ICMA) and Departamento de Física de la Materia Condensada

CSIC - Universidad de Zaragoza

C/ Pedro Cerbuna 12, 50009 Zaragoza (Spain)

E-mail: evange@unizar.es

Homepage: http://molchip.unizar.es/

Dr. J. Sesé

Instituto de Nanociencia de Aragón and Departamento de Física de la Materia Condensada Universidad de Zaragoza

C/ Mariano Esquillor s/n, 50018 Zaragoza (Spain)

S. Cardona-Serra, Prof. E. Coronado

Instituto de Ciencia Molecular (ICMol)

Universidad de Valencia

Catedrático José Beltrán 2, 46980 Paterna (Spain)

E-mail: eugenio.coronado@uv.es

Keywords: polyoxometalates, molecular nanomagnets, gadolinium, magnetic refrigeration, magnetocaloric effect, ultra-low temperatures

Selected molecular nanomagnets have proved to be valid magnetic refrigerants for low temperatures. ${ }^{1}$ At the basis of this application is the magnetocaloric effect (MCE), i.e. the change of the magnetic entropy $\left(\Delta S_{m}\right)$ and related adiabatic temperature following a change of the applied magnetic field $\left(\Delta B_{0}\right)$. In order to efficiently exploit the MCE for widespread applications, this effect should be maximized within the working temperature range of interest. $^{2}$ A common strategy is that of playing with the magnetic interactions since these set the way in which the magnetic entropy is released as a function of temperature, i.e. it changes from a smooth gradual temperature-dependence in the paramagnetic case into a steeper behaviour when the interactions become important. This can be used advantageously to produce a large $\Delta S_{m}$ by means of a limited change in $T$ and/or $B_{0}$ that is a much larger change 
than that produced in the absence of such interactions, yielding an enhanced refrigeration power $\left(=\int \Delta S_{m} d T\right)$. Unfortunately, a drawback inherent to this approach is that the MCE steeply falls to near zero values below the critical temperature $\left(T_{C}\right)$ of a magnetically ordered region, limiting the lowest temperature which can be attained in a process of adiabatic demagnetization. Therefore, if one targets a magnetic refrigerant material capable of achieving millikelvin temperatures, particular attention should be devoted to avoid any source of magnetic interactions. The same holds true for the magnetic anisotropy that should ideally be negligible, since the smaller the anisotropy, the less pronounced are the crystal field effects which, splitting the energy levels, result in MCE maxima at lower temperature. It is worth pointing out that best performing astronomical instruments rely on sensors cooled to $\mathrm{mK}$. In contrast to conventional ${ }^{3} \mathrm{He}-{ }^{4} \mathrm{He}$ dilution refrigerators, ${ }^{3}$ adiabatic demagnetization refrigerators can be operated in a gravity-free environment - a requirement for space-borne missions. Furthermore, over the last few years we have witnessed a rise in the production of security instruments for nuclear detection that has ultimately led to a worldwide shortage of ${ }^{3} \mathrm{He}$. It is then important to secure an efficient alternative to ${ }^{3} \mathrm{He}$-based technology for ultralow temperatures.

Gadolinium is a common constituent element for magnetic refrigerant materials, mainly because its ${ }^{8} S_{7 / 2}$ ground state provides the largest entropy per single ion. Furthermore, its quenched orbital momentum implies that crystal field effects are extremely small. Bulk gadolinium metal undergoes a ferromagnetic ordering below $T_{C} \approx 294 \mathrm{~K}$ and, for the aforementioned reasons, it cannot be exploited for low-temperature refrigeration. Gadolinium sulfate $^{4,5}$ and gadolinium gallium garnet $^{6}$ are well-known low-temperature magnetic refrigerant materials, although they are limited by their magnetic ordering temperatures of 0.18 and $0.9 \mathrm{~K}$, respectively, and so is the recently investigated gadolinium acetate tetrahydrate with $T_{C} \approx 0.2 \mathrm{~K}{ }^{7}$ Likewise, one may expect a relatively large ordering temperature in the case of extended $\mathrm{Gd}^{3+}$-based systems, such as $1 \mathrm{D}$ chains. ${ }^{8}$ Mixed $\mathrm{Gd}^{3+}$ - 
$\mathrm{Mn}^{3+}, \mathrm{Gd}^{3+}-\mathrm{Co}^{2+}$ and $\mathrm{Gd}^{3+}-\mathrm{Ni}^{2+}$ molecular nanomagnets have been considered as magnetic coolers, ${ }^{9-11}$ but they are not suitable for ultra-low temperatures due to the anisotropy induced by the $\mathrm{Mn}^{3+}, \mathrm{Co}^{2+}$ and $\mathrm{Ni}^{2+}$ ions, respectively. Diluted paramagnetic salts not-containing gadolinium, like cerium magnesium nitrate (CMN) and chromic potassium alum (CPA), can achieve mK temperatures favoured by the weak strength of the interactions between the paramagnetic ions. ${ }^{12}$ However, these commercially-employed magnetic refrigerant materials are also characterized by relatively strong magnetic anisotropy and low refrigeration power, which results from the small effective-spin values and spin to volume ratios.

In this communication, we present two novel molecular nanomagnets based on polyoxometalate $(\mathrm{POM})$ salts with general formula $\mathrm{Na}_{9}\left[\mathrm{Gd}\left(\mathrm{W}_{5} \mathrm{O}_{18}\right)_{2}\right] \cdot 35 \mathrm{H}_{2} \mathrm{O}$ (hereafter shortened as $\left.\mathrm{GdW}_{10}\right)$ and $\mathrm{K}_{12}\left(\mathrm{GdP}_{5} \mathrm{~W}_{30} \mathrm{O}_{110}\right) \cdot 54 \mathrm{H}_{2} \mathrm{O}$ (hereafter shortened as $\mathrm{GdW}_{30}$ ), respectively. Both are characterized by having a single $\mathrm{Gd}^{3+}$ ion per molecular unit. ${ }^{13,14}$ Each Gd-complex therefore preserves a relatively large-spin ground state, while having a negligible magnetic anisotropy. Importantly, intermolecular distances are large and each magnetic ion is encapsulated by a closed polyoxowolframate framework, which act as a capping ligand (Figure 1). We shall see below that all this results in an effective screening of the magnetic interactions, since we experimentally observe spin ordering below $\approx 36 \mathrm{mK}$ in case of $\mathrm{GdW}_{10}$ and paramagnetism down to the lowest investigated temperature of $\approx 10 \mathrm{mK}$ in case of $\mathrm{GdW}_{30}$. Our findings make $\mathrm{GdW}_{10}$ and $\mathrm{GdW}_{30}$ appealing for application as magnetic refrigerant materials for ultra-low temperatures.

We investigated the magnetic properties of $\mathrm{GdW}_{10}$ and $\mathrm{GdW}_{30}$ by susceptibility $\left(\chi_{M}\right)$ experiments in the $\approx 10 \mathrm{mK}<T<300 \mathrm{~K}$ range. For both, the room-temperature $\chi_{M} T$ (Figure S1) value is $7.88 \mathrm{~cm}^{3} \mathrm{~K} \mathrm{~mol}^{-1}$ and it stays constant on decreasing the temperature down to $\approx$ $0.1 \mathrm{~K}$, below which it very slightly deviates from the Curie law expected for a spin-only $(g=$ 2.00) uncoupled $\mathrm{Gd}^{3+}$ center (Figure 2). Anticipating the discussion on the heat capacity data, we attribute this deviation to the presence of a small magnetic anisotropy of the molecular 
units, which is nonetheless observable at these very low temperatures. Besides, $\chi_{M}(T)$ of $\mathrm{GdW}_{10}$ has an anomaly centered at $\approx 36 \mathrm{mK}$ that we associate to a magnetic phase transition driven by dipole-dipole interactions, whereas $\mathrm{GdW}_{30}$ is paramagnetic in the whole investigated temperature range (Fig. 2). An explanation of such a different behaviour could be ascribed to the increase of the average intermolecular distance $(r)$ from $\approx 1 \mathrm{~nm}$ to $\approx 2 \mathrm{~nm}$ for $\mathrm{GdW}_{10}$ and $\mathrm{GdW}_{30}$, respectively. ${ }^{15}$ If fact, if we look at the details of the crystal structure, we notice that the shortest Gd-Gd distance is $1.12 \mathrm{~nm}$ in $\mathrm{GdW}_{10}$, while it increases to $1.56 \mathrm{~nm}$ in $\mathrm{GdW}_{30}$ (Figure 1). As the strength of the dipolar interaction decreases with $1 / r^{3}$, we expect the spin ordering temperature to scale accordingly, therefore leading to a value of $36 / 2^{3} \approx 4 \mathrm{mK}$ for $\mathrm{GdW}_{30}$. Magnetization versus field data, collected for the $2 \mathrm{~K}<T<20 \mathrm{~K}$ range and applied fields $B_{0}$ up to $5 \mathrm{~T}$ (see Insets of Fig. 2), corroborate the paramagnetic susceptibility of both complexes in this temperature range. We observe that the isothermal molar magnetization curves saturate to the value of $7 N \mu_{B}$ and their field-dependence is well described by the Langevin function for $s=7 / 2$ and $g=2.00$ (solid line).

Figure 3 depicts the experimental magnetic contribution of the heat capacity $\left(C_{m}\right)$ for $\mathrm{GdW}_{10}$ and $\mathrm{GdW}_{30}$ in the $\approx 0.35 \mathrm{~K}<T<20 \mathrm{~K}$ range for some selected applied fields, as labelled. As anticipated by the $\chi_{M}$ measurements, no sign of a phase transition is detected within the investigated temperature range. The experimental curves in Figs. 3(a) and 3(b) were obtained by subtracting the non-magnetic phonon contribution, arising from thermal vibrations of the lattice, from the total measured heat capacity (Fig. S2). For the sake of completeness, we report that the phonon contribution can be well modelled by a Debye function with $\Theta_{D}=48.9$ $\mathrm{K}$ and $27.7 \mathrm{~K}$ for the Debye temperatures of $\mathrm{GdW}_{10}$ and $\mathrm{GdW}_{30}$, respectively, which are typical values for this class of metal-oxide cluster compounds. ${ }^{16}$ Because $\mathrm{GdW}_{30}$ has larger intermolecular distances, ${ }^{15}$ softer low-energy modes, yielding smaller $\Theta_{D}$, are indeed to be expected. For both, we observe a broad Schottky-type anomaly that shifts towards higher temperatures on increasing applied field and that we associate with the splitting of the $s=7 / 2$ 
multiplet of the Gd molecules. The fact that the zero-field $C_{m}$ also shows such a feature denotes the presence of an internal field locally acting on the $\mathrm{Gd}^{3+}$ ions, either caused by anisotropy or magnetic interactions. Tentatively, we disregard the latter because the weak dipolar interactions should not be effective in this temperature range and on the basis of the experimental observation that the heat capacity remains unaltered by diluting the $\mathrm{Gd}^{3+}$ ions in $\mathrm{Gd}_{0.3} \mathrm{Y}_{0.7} \mathrm{~W}_{10}$ (Fig. S3). Therefore, we fit the experimental curves using the single-spin Hamiltonian $H=D s_{z}^{2}+g \mu_{B} \vec{B} \cdot \vec{s}$, where $D$ is an uniaxial zero-field anisotropy term. We obtain the best fits for $D=-0.18 \mathrm{~K}$ in case of $\mathrm{GdW}_{10}$ and $D=6.5 \cdot 10^{-2} \mathrm{~K}$ in case of $\mathrm{GdW}_{30}$, where the positive sign implies that the $z$-axis is not a preferential spin orientation. ${ }^{17}$ This small axial (planar) anisotropy is well consistent with the molecular symmetry of the $\mathrm{GdW}_{10}$ $\left(\mathrm{GdW}_{30}\right)$ molecule (Figure 1).

The direct comparison in Fig. 3(c) of the zero-field $C_{m}$ for $\mathrm{GdW}_{10}$ and $\mathrm{GdW}_{30}$ with that corresponding to the bulk metal reveals a spectacular effect on the magnetic phase transition, i.e. the sharp $\lambda$-like feature at $T_{C} \approx 294 \mathrm{~K}$. From the heat capacities, the temperature dependence of the magnetic entropies is obtained by integration, i.e. $S_{m}(T)=\int_{0}^{T}\left(C_{m} / T\right) d T$, and depicted in Fig. 3(d). It is seen that the total molar entropy gain that is reached from zero to infinite temperature does not depend on whether we consider the isolated $\mathrm{Gd}^{3+}$ ions or the higher-temperature bulk-ferromagnet, since it corresponds to the maximum gain $R \ln (2 s+1)=$ $2.08 R$ for $s=7 / 2$.

We finally evaluate the MCE for the $\mathrm{GdW}_{10}$ and $\mathrm{GdW}_{30}$ molecular complexes. This procedure includes the calculation from the entropy curves, viz. from the measured heat capacities depicted in Figure 3, of the magnetic entropy change $\Delta S_{m}\left(T, \Delta B_{0}\right)$ for selected field changes $\Delta B_{0}=B_{f}-B_{i}$, where $f$ and $i$ indicate final and initial states, respectively. The so-obtained temperature dependencies of $\Delta S_{m}$ are shown in Figure 4 for several $\Delta B_{0}$ values. One can see 
that, for a maximum field change of $\Delta B_{0}=(7-0) \mathrm{T},-\Delta S_{m}$ reaches a maximum value of 4.7 $\mathrm{Jkg}^{-1} \mathrm{~K}^{-1}$ at $T \approx 1.8 \mathrm{~K}$ and $1.9 \mathrm{Jkg}^{-1} \mathrm{~K}^{-1}$ at $T \approx 1.3 \mathrm{~K}$ for $\mathrm{GdW}_{10}$ and $\mathrm{GdW}_{30}$, respectively. We notice that this $\Delta S_{m}$ value for $\mathrm{GdW}_{30}$ compares well with the full change of the magnetic entropy (2.08R), which indeed corresponds to $1.9 \mathrm{Jkg}^{-1} \mathrm{~K}^{-1}$ considering its molecular weight $\approx$ $9029 \mathrm{~g} / \mathrm{mol}$. In case of $\mathrm{GdW}_{10}$, a field change somewhat larger than $7 \mathrm{~T}$ is needed in order for $-\Delta S_{m}$ to reach its maximum value (2.08R), which in this latter case corresponds to $5.1 \mathrm{Jkg}^{-1} \mathrm{~K}^{-}$ ${ }^{1}$, according to the lighter molecular weight $(\approx 3410 \mathrm{~g} / \mathrm{mol})$. The stronger field dependence at lower temperatures in case of $\mathrm{GdW}_{30}$ is understood taking into account its lower anisotropy. ${ }^{1}$ Although these effects are not extraordinary large, they take place at remarkably low temperatures. This is especially true for $\mathrm{GdW}_{30}$ which, to our knowledge, represents the best realization of a paramagnetic single-atom gadolinium compound.

In conclusion, as the current state-of-the-art in magnetic refrigeration employing molecular nanomagnets did not permit achieving temperatures lower than $\approx 0.2 \mathrm{~K}$ at best, ${ }^{1,7-11}$ we aimed in this work to extend the working temperature range down to temperatures much closer to absolute zero. The herein-investigated Gd-based POMs with formula $\mathrm{GdW}_{10}$ and $\mathrm{GdW}_{30}$, respectively, beautifully reach our goal. In an adiabatic demagnetization, the lowest attainable temperature is limited by the spin anisotropy and ordering temperature. Our experiments in $\mathrm{GdW}_{10}$ and $\mathrm{GdW}_{30}$ down to $\approx 10 \mathrm{mK}$ demonstrate that both limitations can be overcome by chemically engineering the molecules in such a way to effectively screen all magnetic interactions, suggesting their use as coolers for ultra-low temperatures. The inherent downside of such an approach is related to the heavy structural POM framework of each molecular unit that, being non-magnetic, ultimately lowers the cooling power. Indeed, a conventionally employed magnetic refrigerant for $\mathrm{mK}$, such as $\mathrm{CMN}$, provides a magnetic entropy change which can be as large as $R \ln 2$, corresponding to $\approx 7.5 \mathrm{Jkg}^{-1} \mathrm{~K}^{-1}$. ${ }^{12}$ Although this value is still relatively large, its strong dependency on the orientation of the applied field $\left(g_{/ /} / g_{\perp}<0.014\right)$ makes its application less efficient than that of the Gd-based POMs. The search for other 
mononuclear molecular isotropic nanomagnets having lighter capping ligands, yet effective in screening all magnetic interactions, should motivate further studies.

\section{Experimental}

$\mathrm{Na}_{9}\left[\mathrm{Gd}\left(\mathrm{W}_{5} \mathrm{O}_{18}\right)_{2}\right] \cdot 35 \mathrm{H}_{2} \mathrm{O}$ (1) was prepared following a previously described procedure. ${ }^{18} \mathrm{~A}$ total of $8.3 \mathrm{~g}(25 \mathrm{mmol})$ of $\mathrm{Na}_{2} \mathrm{WO}_{4} \cdot 2 \mathrm{H}_{2} \mathrm{O}$ was dissolved in $20 \mathrm{~mL}$ of water. The resulting solution was adjusted to $\mathrm{pH}$ 7.4-7.5 with acetic acid. Subsequently, an aqueous solution (2 $\mathrm{mL}$ ) containing $0.93(2.5 \mathrm{mmol})$ of $\mathrm{GdCl}_{3} \cdot 6 \mathrm{H}_{2} \mathrm{O}$ was added dropwise to the abovementioned solution under continuous stirring and heated up to $85^{\circ} \mathrm{C}$. Crude crystals were isolated when this solution was left to stand at room temperature.

$\mathrm{K}_{12} \mathrm{GdP}_{5} \mathrm{~W}_{30} \mathrm{O}_{110} \cdot 54 \mathrm{H}_{2} \mathrm{O}$ was prepared following a previously described method. ${ }^{19}$ In a typical experiment $\mathrm{K}_{12,5} \mathrm{Na}_{1.5}\left[\mathrm{NaP}_{5} \mathrm{~W}_{30} 0_{110}\right] \cdot 15 \mathrm{H}_{2} \mathrm{O}(1 \mathrm{~g}, 0.12 \mathrm{mmol})$ was dissolved in $12 \mathrm{~mL}$ of water and the solution was heated to $60-70{ }^{\circ} \mathrm{C}$. To this solution two equivalents of the gadolinium ion as the chloride salt dissolved in water $(3 \mathrm{~mL})$ was added dropwise. The mixture was placed in a Parr Model 4746 or Model 4748 sample preparation bomb and heated to $160{ }^{\circ} \mathrm{C}$ overnight. After the solution had cooled to room temperature, the product was isolated by the addition of $4 \mathrm{~g}$ of solid KCI.

Physical characterization. Magnetic measurements down to $2 \mathrm{~K}$ and heat capacity measurements using the relaxation method down to $0.35 \mathrm{~K}$ on powder samples were carried out using commercial setups for the $0<B_{0}<7 \mathrm{~T}$ magnetic field range. Susceptibility measurements below $2 \mathrm{~K}$ were performed using a homemade SQUID-based AC- $\mu$ suceptometer installed in a ${ }^{3} \mathrm{He}-{ }^{4} \mathrm{He}$ dilution fridge. ${ }^{20}$ The latter provided data in arbitrary units, which we did properly scaled in order to match them with the susceptibility data collected with the commercial magnetometer for the overlap $2 \mathrm{~K}<T<4 \mathrm{~K}$ range (Figure S1). In case of the $\mu$-susceptometer, the sample consisted of a collection of small grains of c.a. $10^{-3}$ $\mathrm{mm}^{3}$. 


\section{Acknowledgments}

This work has been supported by MINECO through grants MAT2009-13977 and CSD200700010.

Received: ((will be filled in by the editorial staff))

Revised: ((will be filled in by the editorial staff)) Published online: ((will be filled in by the editorial staff))

_[1] For recent overviews, see: a) M. Evangelisti, E. K. Brechin, Dalton Trans. 2010, 39, 4672-4676, and references therein; b) R. Sessoli, Angew. Chem. 2012, 124, 43-45; Angew. Chem. Int.-Ed. 2012, 51, 43-45, and references therein.

_[2] V. K. Pecharsky, K. A. Gschneidner Jr., J. Magn. Magn. Mater. 1999, 200, 44-56.

_[3] See, e.g., D. S. Betts, An introduction to millikelvin technology, Cambridge University Press, New York, 1989.

[4] a) W. F. Giauque, D. P. MacDougall, Phys. Rev. 1935, 43, 768-768; b) W. F. Giauque, D. P. MacDougall, J. Am. Chem. Soc. 1935, 57, 1175-1185.

_[5] R. F. Wielinga, J. Lubbers, W. J. Huiskamp, Physica 1967, 37, 375-392.

_[6] R. D. McMichael, J. J. Ritter, R. D. Shull, J. Appl. Phys. 1993, 73, 6946-6948.

_[7] M. Evangelisti, O. Roubeau, E. Palacios, A. Camón, T. N. Hooper, E. K. Brechin, J. J. Alonso, Angew. Chem. 2011, 123, 6736-6739; Angew. Chem. Int.-Ed. 2011, 50, 6606-6609.

_[8] F. S. Guo, J. D. Leng, J. L. Liu, Z. S. Meng, M. L. Tong, Inorg. Chem. 2012, 51, 405413.

_[9] a) G. Karotsis, M. Evangelisti, S. J. Dalgarno, E. K. Brechin, Angew. Chem. 2009, 121, 10112-10115; Angew. Chem. Int.-Ed. 2009, 48, 9928-9931; b) G. Karotsis, S. Kennedy, S. J. 
Teat, C. M. Beavers, D. A. Fowler, J. J. Morales, M. Evangelisti, S. J. Dalgarno, E. K. Brechin, J. Am. Chem. Soc. 2010, 132, 12983-12990.

_[10] Y.-Z. Zheng, M. Evangelisti, R. E. P. Winpenny, Chem. Sci. 2011, 2, 99-102.

_[11] a) Y.-Z. Zheng, M. Evangelisti, R. E. P. Winpenny, Angew. Chem. 2011, 123, 37763779; Angew. Chem. Int.-Ed. 2011, 50, 3692-3695; b) Y.-Z. Zheng, M. Evangelisti, F. Tuna, R. E. P. Winpenny, J. Am. Chem. Soc. 2012, 134, 1057-1065.

_[12] See, e.g., R. P. Hudson, Principles and application of magnetic cooling, NorthHolland Publishing Company, Amsterdam, 1972.

_[13] M. A. AlDamen, J. M. Clemente-Juan, E. Coronado, C. Martí-Gastaldo, A. GaitaAriño, J. Am. Chem Soc. 2008, 130, 8874-8875.

_[14] M. A. AlDamen, S. Cardona-Serra, J. M. Clemente-Juan, E. Coronado, A. Gaita-Ariño, C. Martí-Gastaldo, F. Luis, O. Montero, Inorg. Chem. 2009, 48, 3467-3479.

_[15] The compound $\mathrm{GdW}_{10}$ crystallizes in space group triclinic with $a=12.7417(3) \AA, b=$ 13.0815(3) $\AA, c=20.4366(5) \AA$, whereas $\mathrm{GdW}_{30}$ crystallizes in space group monoclinic with $a=17.5255(10) \AA, b=21.0554(2) \AA, c=35.5201(2) \AA$. From the unit cell volumes, we obtain the mean values of the intermolecular separations: $10 \AA$ for $\mathrm{GdW}_{10}$ and $20 \AA$ for $\mathrm{GdW}_{30}$.

_[16] M. Evangelisti, F. Luis, L. J. de Jongh, M. Affronte, J. Mater. Chem. 2006, 16, 25342549.

_[17] We anticipate that preliminary EPR experiments have corroborated the $D$ value estimated from heat capacity data.

_[18] T. Ozeki, T. Yamase, Acta Crystallogr. 1994, C50, 327-330.

_[19] I. Creaser, M. C. Heckel, R. J. Neitz, M. T. Pope, Inorg. Chem. 1993, 32, 1573-1578.

_[20] M. J. Martínez-Pérez, J. Sesé, F. Luis, D. Drung, T. Schurig, Rev. Sci. Instrum. 2010, $81,016108 / 1-3$. 

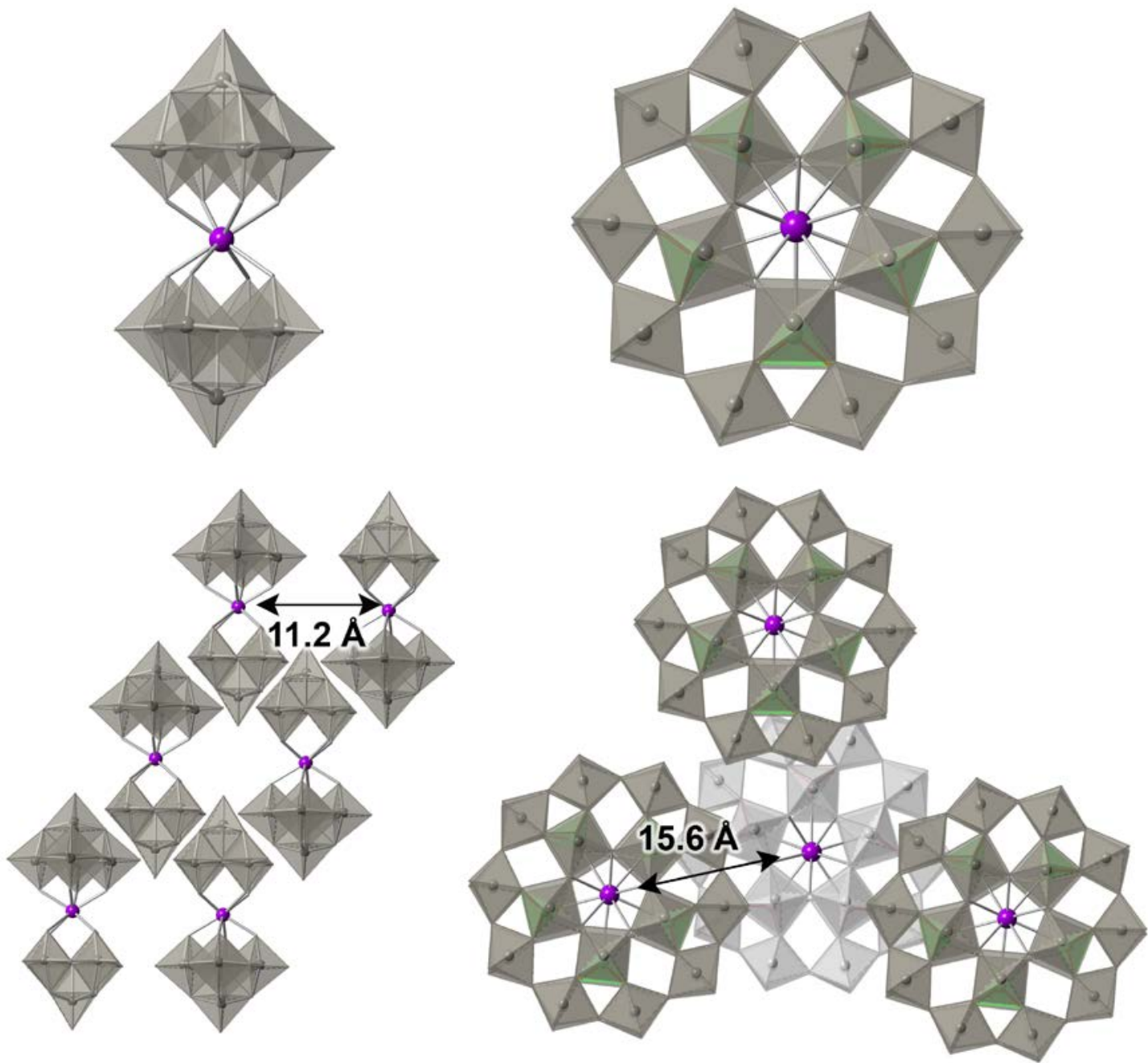

Figure 1. Top: Molecular structures of $\mathrm{GdW}_{10}$ (left) and $\mathrm{GdW}_{30}$ (right). Bottom: Crystal packing of both systems, showing the minimal Gd-Gd distance. Colour code: Gd, purple; $\mathrm{WO}_{6}$, grey. 


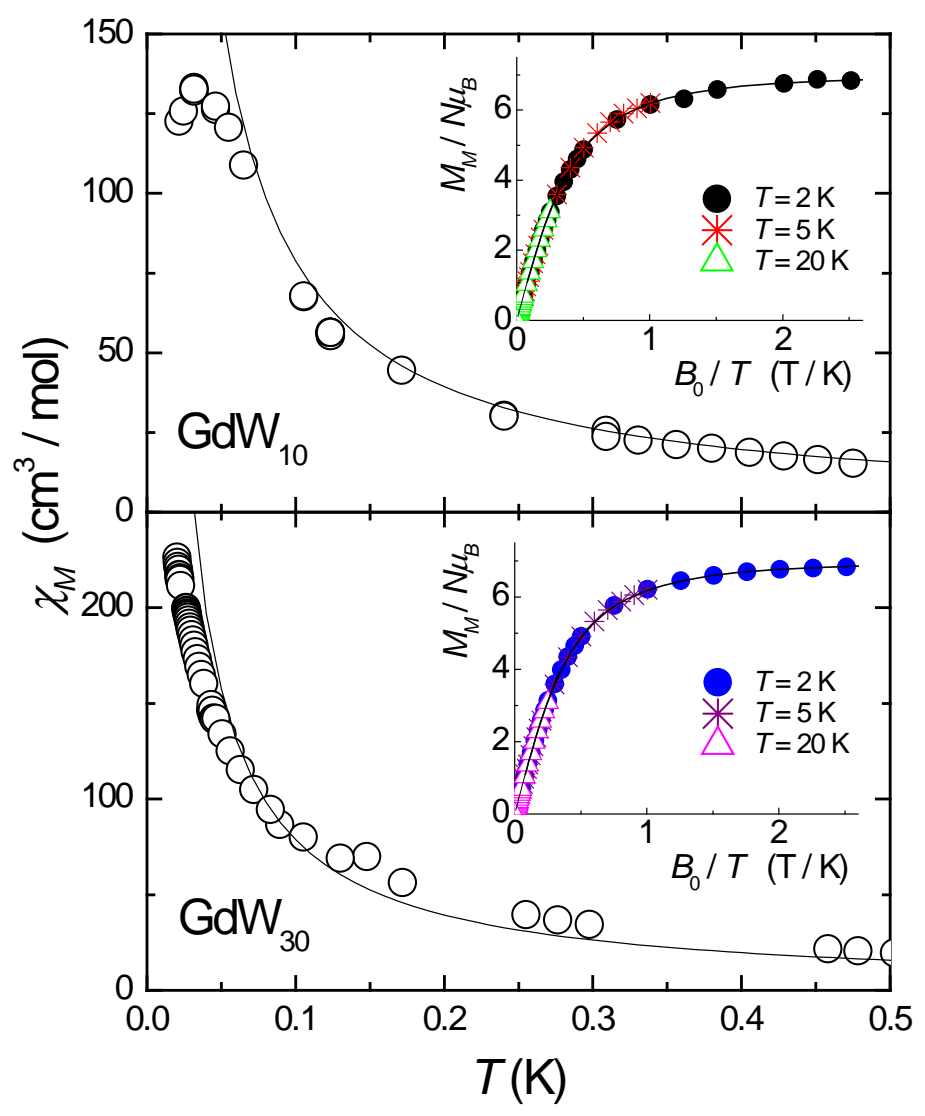

Figure 2. Experimental molar susceptibility of $\mathrm{GdW}_{10}$ (top) and $\mathrm{GdW}_{30}$ (bottom) versus temperature for the lowest investigated temperatures and $B_{0}=0$, together with Curie law for $s$ $=7 / 2$ and $g=2.00$. Inset: experimental reduced molar magnetization $\left(M_{M} / N \mu_{B}\right)$ of $\mathrm{GdW}_{10}$ (top) and $\mathrm{GdW}_{30}$ (bottom) versus $B_{0} / T$ in the ranges $2 \mathrm{~K}<T<20 \mathrm{~K}$ and $0<B_{0}<5 \mathrm{~T}$, as labelled. Full lines are the calculated curves using the Langevin function. 

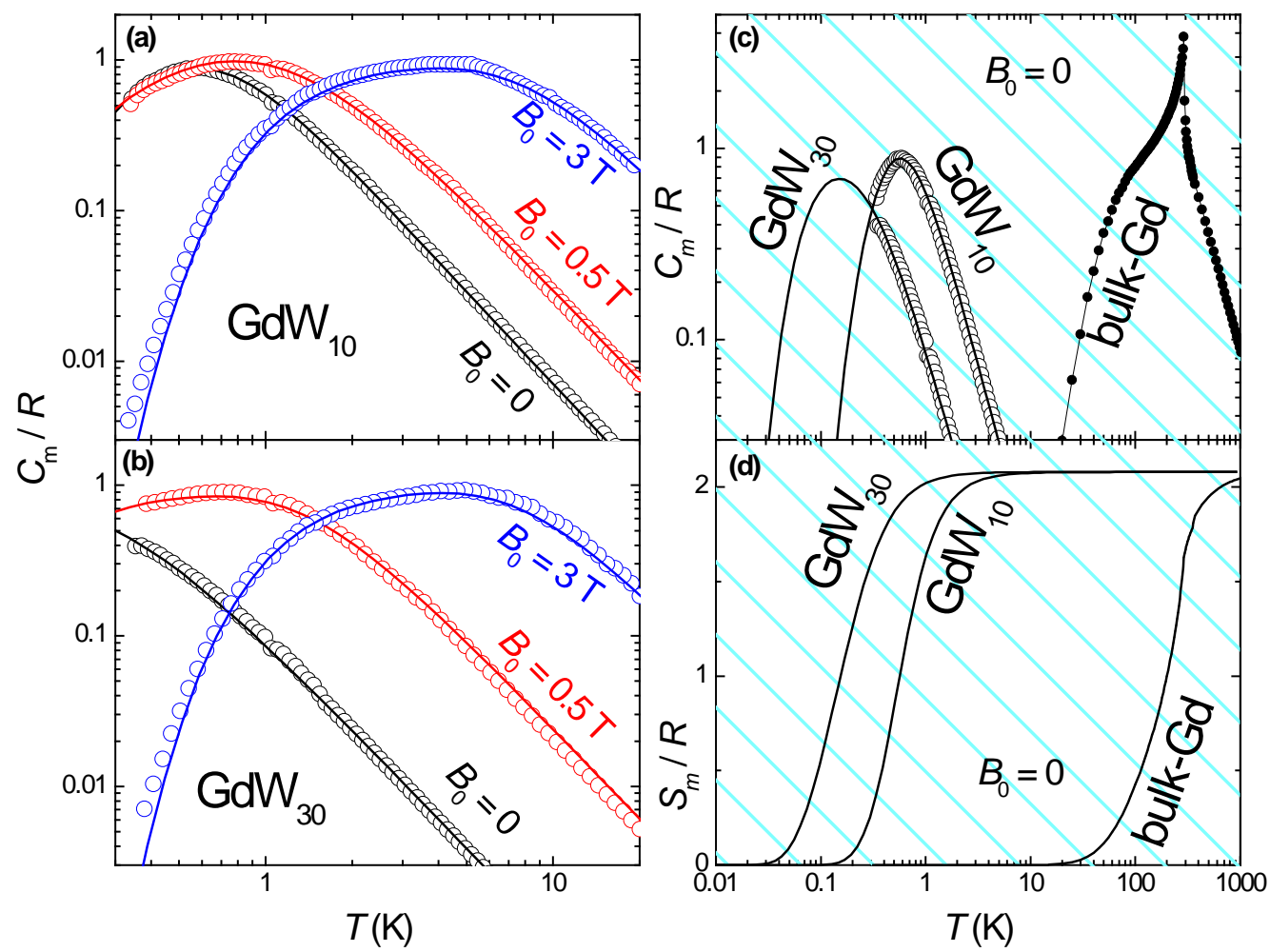

Figure 3. (a)-(b) experimental magnetic heat capacity $\left(C_{m}\right)$ of $\mathrm{GdW}_{10}$ (top) and $\mathrm{GdW}_{30}$ (bottom) normalized to the gas constant $(R)$ versus temperature for some selected applied fields $B_{0}$, as labelled. (c) zero-field $C_{m}$ of bulk-gadolinium together with that of $\mathrm{GdW}_{10}$ and $\mathrm{GdW}_{30}$, for comparison. Solid lines in (a), (b) and (c) are the calculated Schottky heat capacities for $D=0.18 \mathrm{~K}$ in case of $\mathrm{GdW}_{10}$ and $D=-6.5 \cdot 10^{-2} \mathrm{~K}$ in case of $\mathrm{GdW}_{30}$. (d) zerofield magnetic entropy of $\mathrm{GdW}_{10}, \mathrm{GdW}_{30}$ and bulk-gadolinium versus temperature, as obtained from the $C_{m}$ data depicted in (c). 


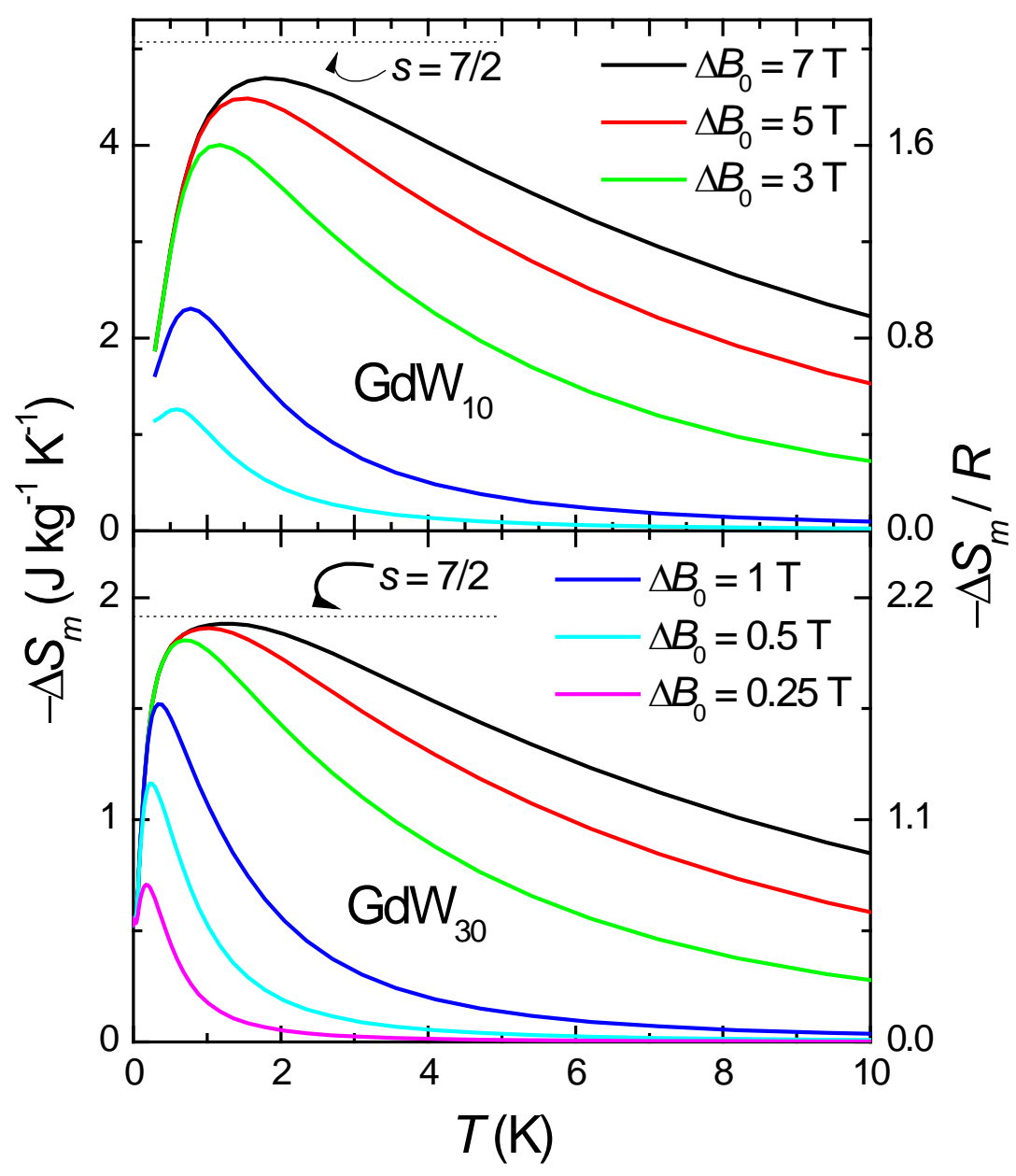

Figure 4. Temperature dependencies of the magnetic entropy change $\Delta S_{m}$ of GdW 10 (top) and $\mathrm{GdW}_{30}$ (bottom) for applied field changes $\Delta B_{0}$, as indicated. The $\Delta S_{m}$ is in units of $\mathrm{Jkg}^{-1} \mathrm{~K}^{-1}$ on the left vertical axis, whilst it is normalized to the gas constant $R$ on the right vertical axis. The dotted line is the full entropy content for an $s=7 / 2$ spin system. 


\section{Table of contents entry.}

The polyoxometalate clusters with formula $\left[\mathrm{Gd}\left(\mathrm{W}_{5} \mathrm{O}_{18}\right)_{2}\right]^{9-}$ and $\left[\mathrm{Gd}\left(\mathrm{P}_{5} \mathrm{~W}_{30} \mathrm{O}_{110}\right)\right]^{12-}$ each carry a single magnetic ion of gadolinium, which is the most widespread element among magnetic refrigerant materials. In an adiabatic demagnetization, the lowest attainable temperature is limited by the presence of magnetic interactions that bring about magnetic order below a critical temperature. We demonstrate that this limitation can be overcome by chemically engineering the molecules in such a way to effectively screen all magnetic interactions, suggesting their use as ultra-low-temperature coolers.

Keywords: polyoxometalates, molecular nanomagnets, gadolinium, magnetic refrigeration, magnetocaloric effect, ultra-low temperatures

Authors: M.-J. Martínez-Pérez, O. Montero, M. Evangelisti,* F. Luis, J. Sesé, S. CardonaSerra, E. Coronado*

Title: Fragmenting gadolinium: Mononuclear polyoxometalate-based magnetic coolers for ultra-low temperatures

ToC figure:

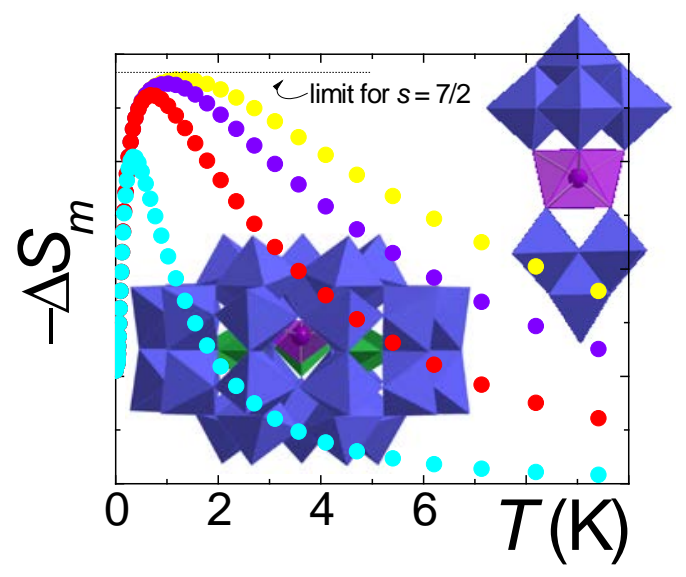


Supporting Information should be included here (for submission only; for publication, please provide Supporting Information as a separate PDF file).

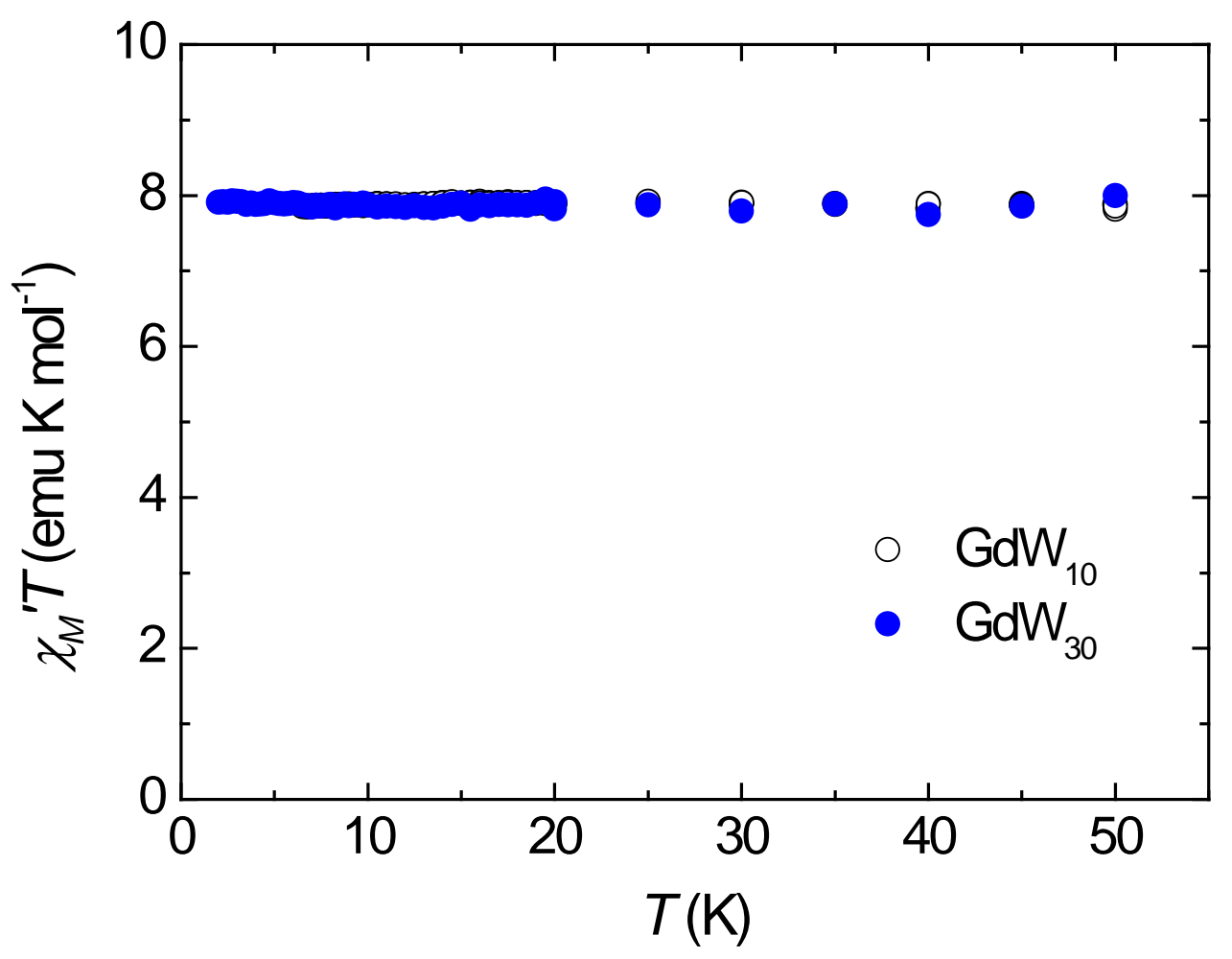

Figure S1. Experimental molar susceptibility $\left(\chi_{M} T\right)$ of $\mathrm{GdW}_{10}$ and $\mathrm{GdW}_{30}$ versus temperature, collected with a commercial AC magnetometer operating at $T>2 \mathrm{~K}$. The in-phase signal is frequency-independent and no out-of-phase signal is detected in the whole investigated temperature range. 


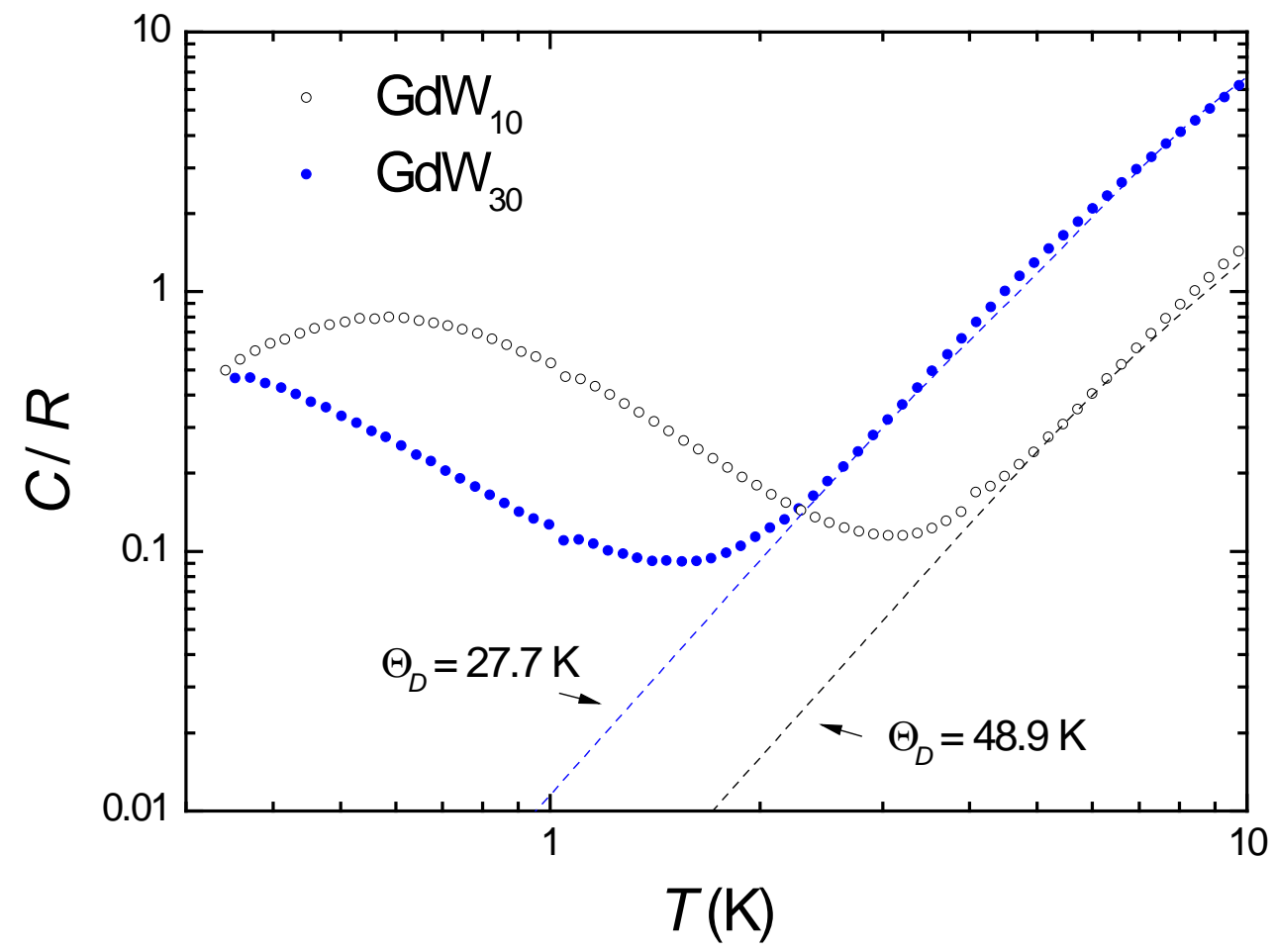

Figure S2. Experimental zero-field heat capacity $(C)$ of $\mathrm{GdW}_{10}$ and $\mathrm{GdW}_{30}$ normalized to the gas constant $(R)$ versus temperature. Dashed lines are the calculated lattice contributions providing $\Theta_{D}=48.9 \mathrm{~K}$ and $27.7 \mathrm{~K}$ for the Debye temperatures of $\mathrm{GdW}_{10}$ and $\mathrm{GdW}_{30}$, respectively. 


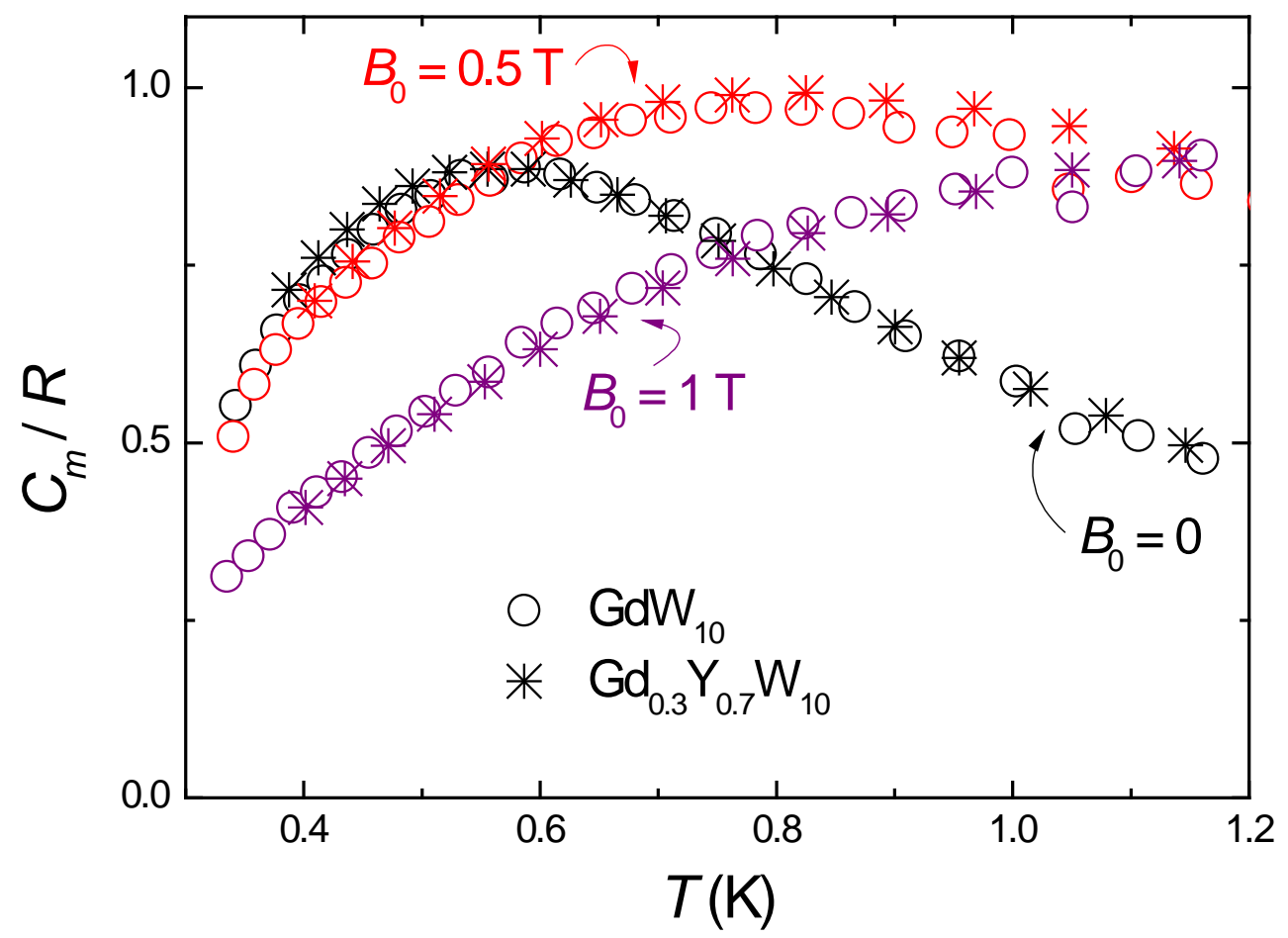

Figure S3. Low-temperature experimental heat capacity normalized to the gas constant for $\mathrm{GdW}_{10}$ and the isostructurally diluted $\mathrm{Gd}_{0.3} \mathrm{Y}_{0.7} \mathrm{~W}_{10}$ for comparison, as a function of temperature and for selected applied magnetic fields, as labelled. 